\title{
Aplicación de modelos de elección discreta regularizados para el análisis de los determi- nantes del consumo cultural en Colombia: el caso de los bienes del patrimonio cultural
}

Aplicação de modelos regularizados de escolha discreta para a análise dos determinantes do consumo cultural na Colômbia: o caso dos ativos do patrimônio cultural

\author{
Andrey David Ramos Ramírez (1) \\ Nora Elena Espinal Monsalve (2) \\ (1) Universidad Nacional de Colombia \\ (2) Universidad Nacional de Colombia
}

\begin{abstract}
This article analyzes the determinants of attendance and frequency of attendance at museums and places of historical interest in Colombia, using data from the Cultural Consumption Survey applied by the $\mathrm{Na}$ tional Administrative Department of Statistics in 2014. Logistic regression models and The Continuation Ratio Model regularized estimated to automatically identify relevant variables to explain cultural consumption decisions, while solving statistical problems associated with the existence of a large number of explanatory variables, such as estimator instability, spurious correlations, incidental multicollinearity and incidental endogeneity, among others. The results indicate that the accumulation of cultural capital is the main determinant of cultural consumption, validating the rational addiction approach in the Colombian context.
\end{abstract}

\section{Keywords}

cultural consumption, cultural heritage, rational dependence, cultural capital, regularized models.

JEL Codes C25, D11, H41, Z11.

\section{Resumen}

En este artículo se analizan los determinantes de la asistencia y frecuencia de asistencia a museos $y$ sitios de interés histórico en Colombia, utilizando los datos de la Encuesta de Consumo Cultural aplicada por el Departamento Administrativo Nacional de Estadística en 2014. Los modelos de regresión logística y el Continuation Ratio Model regularizados que se estiman permiten identificar automáticamente las variables relevantes para explicar las decisiones de consumo cultural, al tiempo que solucionan los problemas estadísticos asociados a la existencia de un número alto de variables explicativas, como es el caso de la inestabilidad de los estimadores, correlaciones espurias, multicolinealidad incidental y endogeneidad incidental, entre otros. Los resultados indican que la acumulación de capital cultural es el principal determinante del consumo cultural, validando así el cumplimiento del enfoque de la adicción racional en el contexto colombiano.

\section{Palabras clave}

consumo cultural, patrimonio cultural, adicción racional, capital cultural, modelos regularizados.

Códigos JEL C25, D11, H41, Z11. 


\section{Introducción}

La literatura empírica en Economía de la Cultura ha prestado especial atención a la "participación" en actividades culturales desde tres puntos de vista: a) asistencia en vivo a presentaciones y espacios culturales, b) consumo a través de medios digitales o c) participación activa como creador o artista (Borgonovi, 2004). Los investigadores se han enfocado más en estudiar la participación a través de la asistencia a eventos culturales en vivo, pues a pesar de las intervenciones del gobierno para impulsar el acceso a este tipo de bienes por medio de subsidios o exenciones fiscales, el número de personas que no participan es alto y persisten disparidades en el consumo entre los diferentes grupos sociales (Ateca-Amestoy, 2009). En este contexto es especialmente interesante identificar los factores que determinan las decisiones de participación cultural con el fin de aportar información útil y precisa para el diseño de políticas incluyentes que contribuyan al cierre de las brechas de participación.

En el presente trabajo se aplican modelos de elección discreta regularizados para identificar los determinantes de la asistencia y frecuencia de asistencia a dos elementos del patrimonio cultural: museos y lugares de interés histórico (monumentos históricos, sitios arqueológicos, monumentos nacionales y centros históricos). Utilizando los datos de la Encuesta de Consumo Cultural aplicada por el Departamento Administrativo Nacional de Estadística (DANE) en 2014 (ECC2014), se estiman las ecuaciones de participación que permiten cuantificar el efecto ceteris paribus de las características sociodemográficas y del capital cultural sobre las decisiones de consumo en las dos actividades. Dada la naturaleza de las variables dependientes, se estiman dos tipos de modelos estadísticos: un modelo logit regularizado para la decisión de asistencia y un Continuation Ratio Model (CRM) regularizado en su formulación backward para la frecuencia de asistencia. El enfoque de la regularización de modelos en este caso de estudio soluciona problemas como la multicolinealidad, inestabilidad de los estimadores, imprecisiones estadísticas, baja capacidad de predicción, entre otros, que se presentan por el alto número de variables explicativas.

El marco teórico de referencia es el de la "adicción racional" propuesto por Stigler y Becker (1977) y Becker y Murphy (1988), el cual plantea que existe cierto tipo de bienes "adictivos" para los cuales la utilidad del consumo presente depende positivamente del consumo pasado, sin que 
esto suponga una excepción a la ley de la utilidad marginal decreciente. De acuerdo con los autores, los bienes adquiridos en el mercado no generan utilidad por sí mismos, sino que, combinados con el tiempo y las características del capital humano, son empleados en la de producción de commodities en el sentido de Becker, de los cuales se obtiene la utilidad. En el caso de los bienes y servicios culturales, por ejemplo, los individuos no obtienen satisfacción del consumo directo sino de la "apreciación cultural" que experimentan, resultado de una función de producción creciente en el tiempo dedicado al consumo y en el capital cultural acumulado por el individuo.

Este enfoque concibe el capital cultural desde un punto de vista microeconómico, como la faceta concreta del capital humano relacionada con las habilidades del individuo para comprender el contenido simbólico, estético e histórico que las actividades culturales incorporan, y que le permite transformar las experiencias culturales en apreciación cultural de una forma más eficiente (Ateca-Amestoy, 2009; Fernández-Blanco y Prieto-Rodríguez, 2009). Desde esta perspectiva, el stock de capital cultural de un individuo puede estar conformado por el nivel de conocimiento, las experiencias de consumo previas y otro tipo de capital físico o humano que contribuyen al conocimiento del bien cultural. Conforme un individuo aumenta su dotación de capital cultural (por ejemplo, a través de inversiones en educación o experiencias de consumo repetidas) las habilidades apreciativas mejoran, el tiempo invertido en el consumo de bienes culturales es más eficiente, y en consecuencia, el nivel de apreciación producida es mayor para un mismo tiempo de consumo. Como resultado, se genera un proceso de adicción racional que en el caso de los bienes culturales es positiva (Stigler y Becker, 1977; Becker y Murphy, 1988).

En el análisis empírico que se realiza en este trabajo se hace énfasis en la relevancia de las variables que determinan el capital cultural para explicar el comportamiento de los individuos con respecto al consumo de bienes culturales. La disponibilidad de información de la ECC2014 permite caracterizar el stock de capital cultural por medio de la educación formal, la práctica cultural activa en talleres, prácticas o actividades lúdicas, y la asistencia en vivo a otras actividades como teatro, galerías de arte y bibliotecas. La hipótesis principal es que las diferencias en la participación cultural están determinadas por las diferencias en las restricciones bajo las cuales toma lugar la elección. Por tanto, una vez se controla por las 
restricciones de tiempo o ingreso, las diferencias en el consumo de bienes culturales en Colombia se explican por diferencias en los niveles de capital cultural acumulado.

Los resultados de esta investigación tienen impacto potencial en la orientación de la política cultural del país, pues permite establecer los factores sociales, culturales o económicos que influyen en las decisiones de consumo de patrimonio de los individuos y que deben ser considerados en el diseño de estrategias que fomenten la participación cultural. Como lo plantean Falk y Katz-Gerro (2016), el entender las características de los individuos que visitan museos y lugares de interés histórico es importante para los estudios sobre estratificación cultural, pues estos sitios hacen parte de un repertorio de cultura "legitimada" asociada a grupos sociales privilegiados, lo que reproduce la desigualdad a partir del capital cultural (Bourdieu, 1984). Adicionalmente, las organizaciones que administran los museos y lugares de interés histórico enfrentan desafíos relacionados con la reducción del presupuesto público, diversificación en el mercado de ocio y nueva competencia del internet (Everett y Barrett, 2009). Por lo tanto, es necesario identificar el perfil de los demandantes de este tipo de actividades para diseñar programas que atraigan visitantes y legitimar así las decisiones de inversión pública y privada en el sector.

El artículo se compone de cinco apartados incluyendo la presente introducción. En el segundo apartado se presenta una revisión de la literatura empírica sobre consumo cultural. En el tercer apartado se describe las fuentes de información, las variables y el enfoque metodológico adoptado en el análisis empírico. En el cuarto apartado se describen los resultados obtenidos en las ecuaciones de participación. En el quinto apartado se discuten los resultados, se presentan las conclusiones y se debate acerca de las perspectivas de investigación en la línea de consumo cultural.

\section{Revisión de la literatura}

En los últimos años un número creciente de estudios empíricos han analizado las decisiones individuales de asistencia a diferentes actividades culturales como: artes escénicas (Borgonovi, 2004; Ateca-Amestoy, 2009; Aguado y Palma, 2012; Wen y Cheng, 2013; Muñiz et al., 2017), museos, galerías de arte y monumentos históricos (Ateca-Amestoy, 2009; Brida et al., 2016; 
Falk y Katz-Gerro, 2016; Muñiz et al., 2017), música (Favaro y Frateschi, 2007; Chan y Goldthorpe, 2007; Guerzoni y Nuccio 2013; Montoro-Pons et al., 2013; Bermúdez et al., 2016), bibliotecas, libros y hábitos de lectura (Fernández-Blanco y Prieto-Rodríguez, 2009; Palma et al., 2014; Notten et al., 2015), cine (Chan y Goldthorpe, 2005; Muñiz et al., 2017), festivales (Devesa et al., 2009; Palma et al., 2013), entre otros. Estos estudios cuantifican el efecto de las características socioeconómicas, el capital cultural o los factores sicológicos de los individuos en las decisiones de asistencia a actividades culturales y/o en la intensidad con que realizan las visitas.

Los resultados encontrados en la literatura indican que la educación es determinante de la participación cultural debido a que el mayor nivel de conocimientos genéricos otorga a los individuos las habilidades y la motivación necesarias para disfrutar de la asistencia a las actividades culturales (Borgonovi, 2004; Ateca-Amestoy, 2008). El ingreso también tiene efectos positivos pues por medio de éste los consumidores pueden asumir los gastos en que incurren durante las visitas (entradas, transporte, recuerdos) (Ateca-Amestoy, 2009; Falk y Katz-Gerro, 2016). Al comparar el efecto de estas dos variables se ha encontrado que en la mayoría de los casos el nivel educativo es un mejor predictor de la participación cultural que el nivel de ingreso (Aguado y Palma, 2012; Muñiz et al., 2017).

La evidencia empírica sugiere una diferencia en el consumo cultural a favor de las mujeres (Borgonovi, 2004; Fernández-Blanco y Prieto-Rodríguez, 2009; Bennet et al., 2013), aspecto que se explica por su mayor sensibilidad frente al arte y la cultura. La edad, por su parte, ha mostrado dos tipos de efectos. Desde el enfoque de adicción racional, los individuos de mayor edad obtienen una utilidad más alta del consumo cultural debido a la acumulación de habilidades y conocimientos sobre los productos culturales durante la vida. En contraste, desde un enfoque de ciclo vital, el efecto de la edad es decreciente en la etapa de entrada en el mercado laboral, luego creciente durante la etapa de estabilidad de ingresos y disponibilidad de tiempo y finalmente decreciente en la etapa de la vejez que viene acompañada de enfermedad y restricciones de movilidad (Borgonovi, 2004). En cuanto a las restricciones de tiempo, la naturaleza de las visitas a actividades culturales las hace especialmente susceptibles de efectos sustitución debido a que la asistencia es tiempo - intensiva y requiere de una planeación para ser disfrutada (Machado et al., 2017); algunas actividades sustitutas pueden ser ver televisión, asistir a otras actividades culturales o 
dedicar más tiempo a las labores diarias como las obligaciones familiares o las obligaciones laborales (Fernández-Blanco y Prieto-Rodríguez, 2009; Muñiz et al., 2017).

Usando datos para Estados Unidos Ateca-Amestoy (2009) demostró que el capital humano del individuo, medido por el nivel de educación formal y la educación específica en artes, es el factor principal para determinar la asistencia a las actividades culturales, aunque no es así para el caso de la frecuencia de asistencia. Un hallazgo similar es reportado por Weng y Cheng (2013) quienes estudiaron la asistencia a artes escénicas en Taiwán. En lo referente a la educación específica en artes, es la educación en la edad adulta la que tiene un mayor efecto sobre la probabilidad y la frecuencia de asistencia (Borgonovi, 2004; Ateca-Amestoy, 2009). En esta misma línea, Fernández-Blanco y Prieto-Rodríguez (2009) encontraron que las dotaciones de capital cultural en todas sus facetas, pero principalmente el acumulado a través de experiencias de consumo cultural, es una pieza fundamental en la formación de las preferencias y en la capacidad de apreciación de los productos culturales, particularmente de la lectura de libros y el uso de bibliotecas.

La mayoría de los estudios de participación revisados han sido aplicados en países desarrollados, principalmente en Estados Unidos y la Unión Europea. En América Latina aún son escasas las contribuciones en esta línea, aunque se destacan los trabajos de Aguado y Palma (2012), Palma et al. (2014); Bermúdez et al. (2016) y Gómez-Hernández y Espinal (2016) aplicados en Colombia; así como Diniz y Machado (2011) y Machado et al. (2017) aplicados en Brasil. Aguado y Palma (2012) y Gómez y Espinal (2016) estudiaron los determinantes de la asistencia a artes escénicas, el primero a nivel nacional y el segundo a nivel regional en Medellín. Estos estudios encontraron que el nivel educativo, la condición socioeconómica y las restricciones de tiempo de los individuos determinan las decisiones de asistencia a artes escénicas. Similar a lo encontrado en los estudios internacionales, el nivel educativo como una variable proxy del capital cultural tiene efecto sobre la participación y la frecuencia de participación, y su efecto marginal es superior al del ingreso.

La elección del método econométrico en estos estudios depende del tipo de variable dependiente. Las decisiones de participación, por ejemplo, se representan por medio de variables binarias que han sido tradicionalmente modeladas a través de modelos de regresión logística o modelos 
probit binomiales (Borgonovi, 2004; Favaro y Frateschi, 2007; Aguado y Palma, 2012; Wen y Cheng, 2013; Gómez y Espinal, 2016). En el trabajo de Guerzoni y Nuccio (2013) la variable dependiente (tipo de música que consume) toma múltiples categorías, y por tanto, se estima un modelo logit multinomial. La frecuencia de participación se representa generalmente por medio de variables categóricas ordenadas o por variables de conteo. En el primer caso los investigadores han optado por modelos logit o probit ordenados (Borgonovi, 2004; Fernández-Blanco y Prieto-Rodríguez, 2009; Aguado y Palma, 2012; Falk y Katz-Gerro, 2016; Gómez y Espinal, 2016), mientras que en el segundo caso se han aplicado modelos de regresión Poisson o modelos binomiales negativos con inflación de ceros (AtecaAmestoy, 2008; Ateca-Amestoy, 2009; Fernández-Blanco y Prieto-Rodríguez, 2009; Devesa et al., 2009; Wen y Cheng, 2013; Montoro-Pons et al., 2013; Palma et al., 2013; Muñiz et al., 2017).

La contribución del presente artículo al acervo de literatura aplicada se puede ver desde dos puntos de vista. En primer lugar, dada la escasa literatura empírica sobre participación cultural en países en vía de desarrollo, este trabajo se convierte en un antecedente para avanzar en la comprensión del comportamiento del consumidor cultural en Colombia y en América Latina. En los trabajos revisados en el país no se encontraron aplicaciones al caso de los museos y los lugares de interés histórico. En segundo lugar, este trabajo propone la aplicación de metodologías de Aprendizaje Estadístico que son de frecuente uso en bioestadística y medicina, pero que no han sido empleadas hasta el momento en la modelación de las decisiones de participación cultural, como es el caso de los modelos de regresión logística regularizados y el CRM regularizado. Estas metodologías permiten obtener resultados más precisos en términos estadísticos en comparación con los métodos tradicionales, que se convierten en información más certera para la formulación de la política cultural.

\section{Metodología}

\subsection{Datos y variables}

Los datos que se utilizan en esta investigación son tomados de la Encuesta de Consumo Cultural (ECC) aplicada por el DANE en el 2014. El objetivo 
de la ECC consiste en "caracterizar las percepciones y formas de comportamiento que expresan las prácticas culturales de la población de 5 años y más" (DANE, 2013:4). En este sentido, la ECC indaga por el consumo cultural de los individuos mayores a 12 años en los componentes de presentaciones y espectáculos, espacios culturales, publicaciones, audiovisuales y música, formación y práctica cultural y hábitos de lectura. Además recoge información sobre las características personales y familiares que permiten un análisis econométrico riguroso.

En el año 2014 se completaron 26.302 cuestionarios sobre una muestra representativa de 8.623 hogares en las cabeceras municipales de las regiones Bogotá, Atlántica, Oriental, Central, Pacífica y Orinoquía-Amazonía. Debido a que el diseño muestral de la ECC es probabilístico, estratificado, multietápico y de conglomerados, se garantiza que la muestra es representativa de la población objeto de análisis. En el presente estudio se usan solamente las 8.623 observaciones de los jefes de hogar considerando que la participación cultural es una actividad típicamente social en la cual el comportamiento de personas cercanas afecta las decisiones de consumo (Ateca-Amestoy, 2009), y por tanto, al incluir todas las observaciones se generarían problemas de no independencia.

El objetivo es encontrar el efecto ceteris paribus de la dotación de capital cultural del individuo sobre la decisión y la intensidad de la asistencia a museos y lugares de interés histórico, con el fin de validar el cumplimiento del enfoque de la adicción racional en el contexto colombiano. Dada la disponibilidad de información de la ECC, el capital cultural es medido en tres dimensiones que han sido utilizadas frecuentemente en la literatura (Ateca-Amestoy, 2009; Fernández-Blanco y Prieto-Rodríguez, 2009): a) el nivel de educación formal, b) la participación activa en prácticas culturales, talleres culturales y actividades lúdicas, y c) la participación pasiva como asistente a otras actividades culturales (teatro, conciertos, bibliotecas, galerías de arte, museos y/o lugares de interés histórico).

Además de introducir las variables del capital cultural, se controla por las variables socioeconómicas que definen las restricciones de tiempo e ingreso a las que se enfrenta el consumidor, así como efectos de dotación por la ubicación geográfica. La especificación empírica que se estima está dada por:

$$
y_{i}=f\left(\alpha+\delta P_{i}+\gamma S_{i}+\tau K_{i}+\lambda R_{i}+\varepsilon_{i}\right),
$$


donde $y_{i}$ es la variable dependiente de decisión o frecuencia de asistencia, $P_{i}$ son las características personales (Edad, Sexo, Estado civil y Etnia), $S_{i}$ son las características socioeconómicas (Ingreso, Ocupación, Personas en el hogar), $K_{i}$ son las características del capital cultural (Nivel educativo, participación activa y participación pasiva) y $R_{i}$ es la ubicación geográfica. En la Tabla 1 se presenta una descripción de las variables empleadas en el análisis.

Tabla 1 Descripción de las variables empleadas en el análisis

\begin{tabular}{|c|c|c|}
\hline Variable & Descripción & Tipo / Categorías \\
\hline \multicolumn{3}{|c|}{ Variables dependientes } \\
\hline Asistencia a museos & $\begin{array}{l}\text { El encuestado asistió a museos en el año } \\
\text { anterior a la encuesta. }\end{array}$ & $\begin{array}{l}\text { Binaria: } \\
\text { 1: Sí } \\
\text { 0: No }\end{array}$ \\
\hline $\begin{array}{l}\text { Frecuencia de } \\
\text { asistencia a museos }\end{array}$ & $\begin{array}{l}\text { Frecuencia de asistencia de los encuesta- } \\
\text { dos que sí asistieron a museos en el año } \\
\text { anterior a la encuesta }\end{array}$ & $\begin{array}{l}\text { Categórica ordenada: } \\
\text { 1: Ocasionalmente } \\
\text { 2: Frecuentemente } \\
\text { 3: Muy frecuentemente }\end{array}$ \\
\hline $\begin{array}{l}\text { Asistencia a lugares } \\
\text { de interés histórico }\end{array}$ & $\begin{array}{l}\text { El encuestado asistió a lugares de interés } \\
\text { histórico en el año anterior a la encuesta. }\end{array}$ & $\begin{array}{l}\text { Binaria: } \\
\text { 1: Sí } \\
\text { 0: No }\end{array}$ \\
\hline $\begin{array}{l}\text { Frecuencia de } \\
\text { asistencia a lugares } \\
\text { de interés histórico }\end{array}$ & $\begin{array}{l}\text { Frecuencia de asistencia de los encuesta- } \\
\text { dos que sí asistieron a lugares de interés } \\
\text { histórico en el año anterior a la encuesta }\end{array}$ & $\begin{array}{l}\text { Categórica ordenada: } \\
\text { 1: Ocasionalmente } \\
\text { 2: Frecuentemente } \\
\text { 3: Muy frecuentemente }\end{array}$ \\
\hline \multicolumn{3}{|l|}{ Variables explicativas } \\
\hline Características Pers & rales & \\
\hline
\end{tabular}

Categórica:

$\begin{array}{ll}\text { Edad } \quad \text { Rango de edad del encuestado } & \text { 1: } 12-24 \text { años } \\ & \text { 2: } 25-60 \text { años } \\ & \text { 3: Más de } 60 \text { años }\end{array}$

Categórica:

Sexo Sexo del encuestado 1: Femenino

0 : Masculino

Categórica:

1: Soltero

Estado Civil

Estado civil del encuestado

2: Casado

3: Unión libre

4: Separado o divorciado

5: Viudo 
Tabla 1 (continuación)

\begin{tabular}{|c|c|c|}
\hline Variable & Descripción & Tipo / Categorías \\
\hline Etnia & $\begin{array}{l}\text { El encuestado se considera indígena, } \\
\text { negro, gitano, raizal o palenquero }\end{array}$ & $\begin{array}{l}\text { Binaria: } \\
\text { 1: Sí } \\
0: \text { No }\end{array}$ \\
\hline \multicolumn{3}{|c|}{ Características Socioeconómicas } \\
\hline Ingreso & $\begin{array}{l}\text { Nivel de ingresos mensual declarado por } \\
\text { el encuestado }\end{array}$ & $\begin{array}{l}\text { Categórica: } \\
\text { 1: Entre } 0 \text { y } 1 \text { SMMLV* } \\
\text { 2: Entre } 1 \text { y } 2 \text { SMMLV } \\
\text { 3: Entre } 2 \text { y } 3 \text { SMMLV } \\
\text { 4: Entre } 3 \text { y } 4 \text { SMMLV } \\
\text { 5: Entre } 4 \text { y } 6 \text { SMMLV } \\
\text { 6: Más de } 6 \text { SMMLV }\end{array}$ \\
\hline Ocupación & $\begin{array}{l}\text { Actividad económica en la que el encues- } \\
\text { tado ocupó la mayor parte del tiempo la } \\
\text { semana anterior a la encuesta }\end{array}$ & $\begin{array}{l}\text { Categórica: } \\
\text { 1: Trabajador } \\
\text { 2: Desempleado } \\
\text { 3: Estudiante } \\
\text { 4: Oficios del hogar } \\
\text { 5: Incapacitado permanente } \\
\text { 6: Otra actividad }\end{array}$ \\
\hline Personas & Número de personas que viven en el hogar & Conteo \\
\hline
\end{tabular}

\begin{tabular}{|c|c|c|}
\hline \multicolumn{3}{|c|}{ Características del Capital Cultural } \\
\hline Nivel educativo & $\begin{array}{l}\text { Máximo nivel educativo alcanzado por } \\
\text { el encuestado }\end{array}$ & $\begin{array}{l}\text { Categórica: } \\
\text { 1: Ninguno } \\
\text { 2: Primaria } \\
\text { 3: Secundaria } \\
\text { 4: Media } \\
\text { 5: Superior (Técnica, } \\
\text { Tecnológica, Universitaria) } \\
\text { 6: Posgrado (Especialización, } \\
\text { Maestría, Doctorado) }\end{array}$ \\
\hline Práctica cultural & $\begin{array}{l}\text { El encuestado realizó práctica prácticas } \\
\text { culturales en el año anterior a la encuesta }\end{array}$ & \multirow{4}{*}{$\begin{array}{l}\text { Binarias: } \\
\text { 1: Sí } \\
\text { 0: No }\end{array}$} \\
\hline Talleres culturales & $\begin{array}{l}\text { El encuestado realizó talleres culturales en } \\
\text { el año anterior a la encuesta }\end{array}$ & \\
\hline Actividades lúdicas & $\begin{array}{l}\text { El encuestado asistió a actividades lúdicas } \\
\text { en el año anterior a la encuesta }\end{array}$ & \\
\hline $\begin{array}{l}\text { Asistencia a teatro, } \\
\text { danza u opera }\end{array}$ & $\begin{array}{l}\text { El encuestado asistió a teatro, danza u } \\
\text { opera en el año anterior a la encuesta }\end{array}$ & \\
\hline
\end{tabular}


Tabla 1 (continuación)

\begin{tabular}{|c|c|c|}
\hline Variable & Descripción & Tipo / Categorías \\
\hline $\begin{array}{l}\text { Asistencia } \\
\text { a conciertos }\end{array}$ & $\begin{array}{l}\text { El encuestado asistió a conciertos en el } \\
\text { año anterior a la encuesta }\end{array}$ & \multirow{3}{*}{$\begin{array}{l}\text { Binarias: } \\
\text { 1: Sí } \\
0 \text { : No }\end{array}$} \\
\hline $\begin{array}{l}\text { Asistencia } \\
\text { a bibliotecas }\end{array}$ & $\begin{array}{l}\text { El encuestado asistió a bibliotecas en el } \\
\text { año anterior a la encuesta }\end{array}$ & \\
\hline $\begin{array}{l}\text { Asistencia } \\
\text { a galerías }\end{array}$ & $\begin{array}{l}\text { El encuestado asistió a galerías de arte en } \\
\text { el año anterior a la encuesta }\end{array}$ & \\
\hline \multicolumn{3}{|c|}{ Ubicación geográfica } \\
\hline Región & Región en la que reside el encuestado & $\begin{array}{l}\text { Categórica: } \\
\text { 1: Bogotá } \\
\text { 2: Atlántica } \\
\text { 3: Oriental } \\
\text { 4: Central } \\
\text { 5: Pacíica } \\
\text { 6: Amazonía }\end{array}$ \\
\hline
\end{tabular}

Fuente: Elaboración propia

SMMLV: Salario Mínimo Mensual Legal Vigente para el año 2014, fijado en \$616.000 COP (\$308 USD).

\subsection{Modelos econométricos}

La elección de los modelos econométricos está condicionada por la estructura y disponibilidad de los datos de la ECC. En el caso de la decisión de asistencia, la variable dependiente es binaria: toma el valor de $y_{i}=1$ si el individuo asiste a la actividad y $y_{i}=0$ en otro caso. Por tanto es posible estimar modelos de regresión logística (Cameron y Trivedi, 2005). La frecuencia de asistencia, por su parte, es una variable categórica ordenada con tres posibles alternativas que indican la intensidad con que los asistentes acuden a las actividades en el año anterior a la aplicación de la encuesta: 1) Ocasionalmente (asiste una o dos veces al año), 2) Frecuentemente (asiste entre tres y once veces al año) y 3) Muy frecuentemente (asiste más de doce veces años). Esta variable es modelada por medio de un CRM, asumiendo que la intensidad de las visitas es un fenómeno progresivo que crece a través del tiempo. En efecto, desde el punto de vista de Becker y Murphy (1988), los bienes son adictivos debido a que el consumo pasado incrementa la utilidad marginal del consumo presente. Por tanto, si un individuo actualmente asiste con una frecuencia alta, es porque ha experi- 
mentado un proceso de formación del gusto que lo ha llevado a incrementar progresivamente la intensidad de las visitas como consecuencia de la acumulación de capital cultural y de las sucesivas experiencias.

Para una revisión técnica más profunda de los modelos de regresión logística consultar a Cameron y Trivedi (2005), Hensher et al. (2005), y para el CRM consultar a Hardin et al. (2007) y Hou y Archer (2015).

\subsection{El problema de la alta dimensionalidad y regularización de modelos}

La aplicación de los modelos econométricos requiere la selección de las variables independientes relevantes, es decir, aquellas con la mayor capacidad para explicar y predecir la respectiva variable dependiente. Cuando la dimensión del vector de variables regresoras es alta, la selección de variables se vuelve un proceso complejo y se generan problemas en el análisis estadístico que afectan el desempeño de los modelos estadísticos. Concretamente, la inclusión de un número alto de variables explicativas tiene consecuencias como la acumulación de ruido, correlaciones espurias, heterogeneidad, endogeneidad incidental, multicolinealidad incidental, entre otros, que afectan sensiblemente la inferencia estadística y generan inestabilidades en los estimadores (Fan et al., 2014). En los modelos de elección discreta se genera acumulación de errores de estimación debido a que las reglas de clasificación dependen de un alto número de parámetros asociados a muchos regresores que no contribuyen a la reducción del error de clasificación (Gallón y Vásquez, 2014).

En el contexto del problema que se aborda en esta investigación, el vector de variables explicativas tiene una dimensión relativamente alta si se cuentan las variables dummy creadas para las variables categóricas. Esto afecta la parsimonia del modelo y la interpretación, pues analizar el signo y la significancia de cada uno de los parámetros se vuelve una tarea tediosa. Además, incluir simultáneamente covariables como la educación y el ingreso genera problemas de multicolinealidad debido a que la educación es un determinante de la productividad y por ende de los salarios (Muñiz et al., 2017). Como la inclusión de muchas variables explicativas que además son redundantes afecta negativamente la inferencia estadística, es necesario realizar un proceso de selección de variables riguroso que permita 
obtener resultados precisos y estables, así como modelos parsimoniosos y fáciles de interpretar.

En la literatura estadística se han desarrollado múltiples métodos de selección de variables en modelos de elección discreta. Una de las técnicas más empleadas en la actualidad es la penalización o regularización, la cual consiste en imponer penalidades sobre el vector de coeficientes con el fin de reducir su magnitud, reducir su varianza e identificar automáticamente cuáles coeficientes son diferentes de cero, lo cual implica que la variable asociada es relevante para explicar y predecir la variable dependiente (Hastie et al., 2009; Hastie et al., 2015). La función de penalidad $P_{\alpha}(\beta)$ que se adopta en este trabajo es la Elastic Net, que se obtiene a partir de la combinación lineal convexa entre la penalidad lasso (norma $\ell_{1}$ de $\beta$ ) y la penalidad ridge (norma $\ell_{2}$ de $\beta$ )). Formalmente la penalidad Elastic Net se escribe como (Buhlmann y Van de Geer, 2011):

$$
P_{a}(\beta)=\lambda\left[\frac{1}{2}(1-\alpha) \sum_{j=1}^{p} \beta_{j}^{2}+a \sum_{j=1}^{p}\left|\beta_{j}\right|\right],
$$

donde $\lambda \geq 0$ es el parámetro de regularización que determina qué tan estricta es la penalidad y $\alpha$ es un parámetro de afinamiento que indica la importancia que tiene cada una de las penalidades (lasso o ridge) en el problema de optimización. Conforme $\lambda$ crece, mayor es la restricción sobre el número de variables a incluir y más fuerte es la reducción de magnitud de los coeficientes.

La elección de la penalidad Elastic Net es conveniente pues combina ventajas tanto del método lasso como del método ridge. Por un lado, la geometría de la penalidad lasso permite que la estimación puntual de algunos coeficientes sea exactamente cero (0), facilitando la selección de variables relevantes. Sin embargo, cuando existen variables altamente correlacionadas, el método lasso tiende a seleccionar una de ellas y a excluir la otra (Buhlmann y Van de Geer, 2011). Aunque esto es deseable desde el punto de vista de la parsimonia, genera problemas en la interpretación debido a que en muchas ocasiones es de interés conocer efecto de las dos variables correlacionadas sin perder completamente la información de una de ellas; además incrementa la probabilidad de incurrir en sesgo de variable omitida. La penalidad Elastic Net limita la capacidad del método lasso combinándolo con el método ridge, de manera que los modelos sean estabilizados sin desechar información sobre variables relevantes. 


\subsection{Modelos de elección discreta regularizados}

La función de penalidad se aplica sobre el negativo del logaritmo de la función de log-verosimilitud. En el modelo de regresión logística la estimación de los parámetros se obtiene maximizando la función log-verosímil regularizada (Hastie et al., 2015):

$$
\operatorname{LnL}\left(\alpha, \lambda, \beta \mid x_{i}\right)=\left\{\sum_{i=1}^{n} y_{i} x_{i}^{T} \beta-\ln \left(1+\exp \left(x_{i}^{T} \beta\right)\right]-\lambda P_{\alpha}(\beta)\right\},
$$

donde $x_{i}$ es el vector de variables explicativas y $\beta$ es el vector de parámetros.

En el caso del CRM la función objetivo es (Hou y Archer, 2015):

$$
\operatorname{LnL}\left(\alpha, \lambda, \beta \mid x_{i}\right)=\left\{\sum_{i=1}^{N} \sum_{j=2}^{C}\left(y_{i j} \delta_{i j}+\left(1-\sum_{k=j}^{c} y_{i k}\right)\left(1-\delta_{i j}\right)\right)-\lambda P_{\alpha}(\beta)\right\}
$$

donde $x_{i}$ es el vector de variables explicativas, $\alpha_{c}$ define los $C-1$ thresholds, $\beta$ es el vector de parámetros y $\delta_{i j}$ son las probabilidades de elección para cada una de las alternativas ordenadas.

Para cada valor de $\lambda$ existe un vector de parámetros estimados que maximizan las funciones de log-verosimilitud regularizadas, lo que permite construir trayectorias para los coeficientes que se van acercando a cero conforme $\lambda$ aumenta. Para seleccionar el valor $\lambda$ óptimo que garantiza el equilibrio entre el ajuste del modelo y su estabilidad, se realiza un ejercicio de validación cruzada (Bühlmann, y Van De Geer, 2011) tomando aleatoriamente el $80 \%$ de los datos como entrenamiento y el $20 \%$ como prueba. En el modelo de regresión logística se obtienen dos tipos de valores óptimos: $\lambda_{\text {min }}$ que minimiza el error de clasificación y $\lambda_{1 s e}$ que es el valor más grande de $\lambda$ para el cual el error de clasificación está a un error estándar del valor mínimo. En el CRM se seleccionan también dos valores: $\lambda_{\text {aic }}$ que minimiza el criterio de información AIC y $\lambda_{b i c}$ que minimiza el criterio de información BIC. El valor del parámetro $\alpha$ se fija a criterio del investigador en 0.5 , de modo que la penalidad ridge y la penalidad lasso tengan la misma importancia. La estimación se lleva a cabo usando los paquetes glmnet (Friedman et al., 2010) y glmnetcr (Archer, 2014), desarrollados en el lenguaje R y disponibles bajo licencia pública. 


\section{Resultados}

La información de la ECC2014 arroja que el 10,72\% de los jefes de hogar entrevistados asisten a museos, de los cuales el 79,67\% lo hace ocasionalmente, el $12 \%$ frecuentemente y el $8,32 \%$ muy frecuentemente. La tasa de participación para los sitios de interés histórico es del 18,18\%. El 75,44\% de los asistentes lo hace ocasionalmente, el 12,88\% frecuentemente y el 11,67\% muy frecuentemente. A continuación se describen los resultados de los modelos de elección discreta regularizados que permiten cuantificar las probabilidades de pertenecer a los grupos de asistencia y frecuencia de asistencia.

En la Figura 1 y en la Figura 2 se presenta la trayectoria de los coeficientes para los diferentes valores del parámetro de regularización $\lambda$ (panel derecho) y el error de clasificación asociado (panel izquierdo) de los modelos de regresión logística estimados para la asistencia a museos y la asistencia a lugares de interés histórico, respectivamente. El número de variables relevantes en el modelo para la asistencia a museos es de 29 cuando se considera el parámetro $\lambda_{\min } \mathrm{y}$ de 7 cuando se considera el parámetro $\lambda_{1 s e}$; en ambos casos el error de clasificación se encuentra alrededor del 8,3\%. Similarmente, el número de variables relevantes en el modelo para la asistencia a lugares de interés históricos es de 24 con el parámetro $\lambda_{\min }$ y de 15 con el parámetro $\lambda_{1 s e}$; el error de clasificación es superior al 14,5\%. La Tabla 2 contiene la estimación puntual de los coeficientes para cada valor óptimo de $\lambda$ y los efectos marginales.

En lo que se refiere a las variables relevantes para explicar las decisiones de consumo cultural, los resultados obtenidos son similares a los reportados por estudios aplicados en contextos económicos, sociales, geográficos y culturales diferentes (Borgonovi, 2004; Ateca-Amestoy, 2009; Falk y Katz-Gerro, 2016; Muñiz et al., 2017). La principal diferencia tiene que ver con los efectos marginales de las variables explicativas que en este caso son significativamente menores. Esto se debe a que los modelos regularizados encogen la magnitud de los coeficientes en comparación con los enfoques tradicionales y a que las tasas de participación en las actividades analizadas son más bajas en Colombia y como consecuencia el efecto marginal medio también es menor (Cameron y Trivedi, 2005). 
Figura 1 Selección del parámetro de regularización (Asistencia a museos)
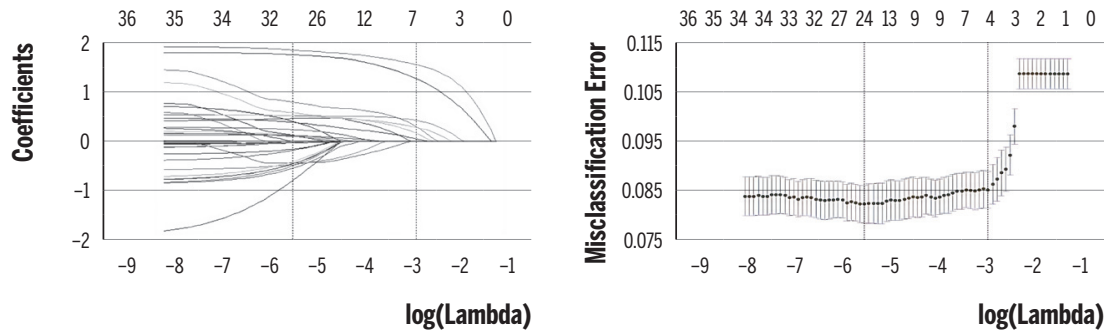

Fuente: Elaboración propia.

Figura 2 Selección del parámetro de regularización (Asistencia a lugares de interés histórico)
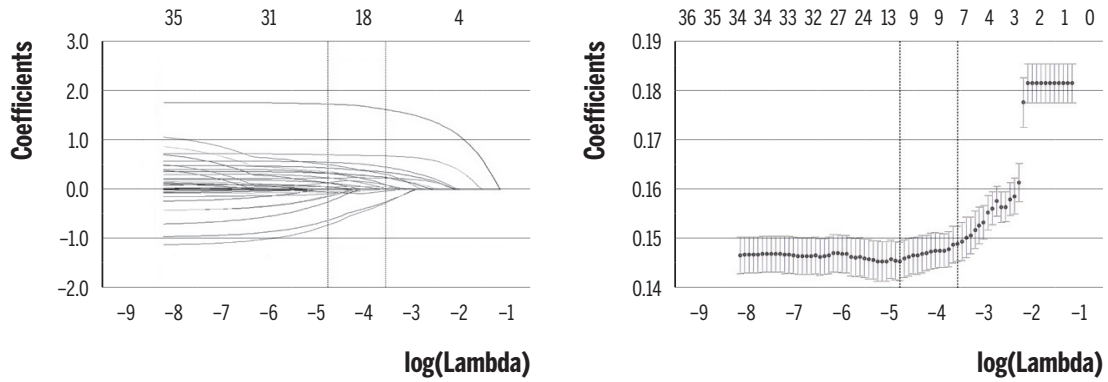

Fuente: Elaboración propia.

Los resultados al considerar el valor de penalización $\lambda_{\text {min }}$ indican que la dotación del capital cultural del individuo es relevante para explicar la decisión de asistencia a museos y lugares de interés histórico en Colombia. En los dos modelos se observa que el efecto del nivel educativo sobre la probabilidad de participación es positivo y monótonamente creciente a partir del nivel de formación Media, lo que se infiere por el incremento en la magnitud de los coeficientes y de los efectos marginales cuando se avanza a niveles más altos de formación. Concretamente, con respecto a quienes no tienen estudios, la probabilidad de asistir a museos es 5,57\% más alta para los individuos con educación superior y 7,63\% para los que tienen posgrado. Los efectos marginales sobre la probabilidad de asistencia a lugares de interés histórico son similares: 5,31\% para los que tienen educación superior y 7,49\% para los que tienen posgrado. 
La participación pasiva a través de la asistencia a teatro danza u ópera, conciertos, bibliotecas y galerías de arte, también es relevante y el efecto es positivo sobre las variables dependientes. Se destaca que los individuos que asisten a galerías de arte tienen 17,68\% más de probabilidad de visitar museos y 10,43\% de visitar lugares de interés histórico. Se observa además una alta complementariedad entre las dos actividades analizadas debido a que el efecto marginal de la asistencia a lugares de interés histórico sobre la asistencia a museos es de $16,77 \%$, mientras que el efecto marginal de la asistencia a museos sobre la asistencia a lugares de interés histórico es de 25,59\%. La participación activa en prácticas culturales, talleres culturales y actividades lúdicas, por su parte, es más importante para determinar la decisión de asistencia a lugares de interés histórico que a museos. El efecto marginal sobre la probabilidad de asistir a museos es de $1,49 \%$ para la práctica cultural, $0,12 \%$ para los talleres culturales y 1,16\% para las actividades lúdicas, mientras que el efecto de estas mismas variables sobre la asistencia a lugares de interés histórico es de 4,86\%, 1,96\% y $8,16 \%$ respectivamente.

De los resultados anteriores es necesario comentar dos aspectos. Primero, aunque la práctica activa y la práctica pasiva en otras actividades culturales compiten con la asistencia a museos y lugares de interés histórico en la asignación del tiempo de ocio, la evidencia muestra una relación de complementariedad que se explica por la mayor exposición de los individuos a los bienes del sector cultural, que mejora las capacidades de apreciación y la eficiencia en el tiempo dedicado al consumo. Este hallazgo apunta a la existencia de un consumidor cultural omnívoro que disfruta de una amplia variedad de productos culturales. Segundo, el efecto del capital cultural, y en específico, del nivel educativo, es un efecto ceteris paribus que recoge la importancia de la formación académica en la adquisición de habilidades cognitivas y de conocimientos que le permiten al individuo apreciar el valor simbólico e histórico que las actividades del sector cultural incorporan. La educación también podría influir indirectamente a través del canal de los ingresos (Favaro y Frasteschi, 2007), sin embargo, como en este trabajo el nivel de ingresos se considera explícitamente como una variable independiente, éste efecto es controlado. 


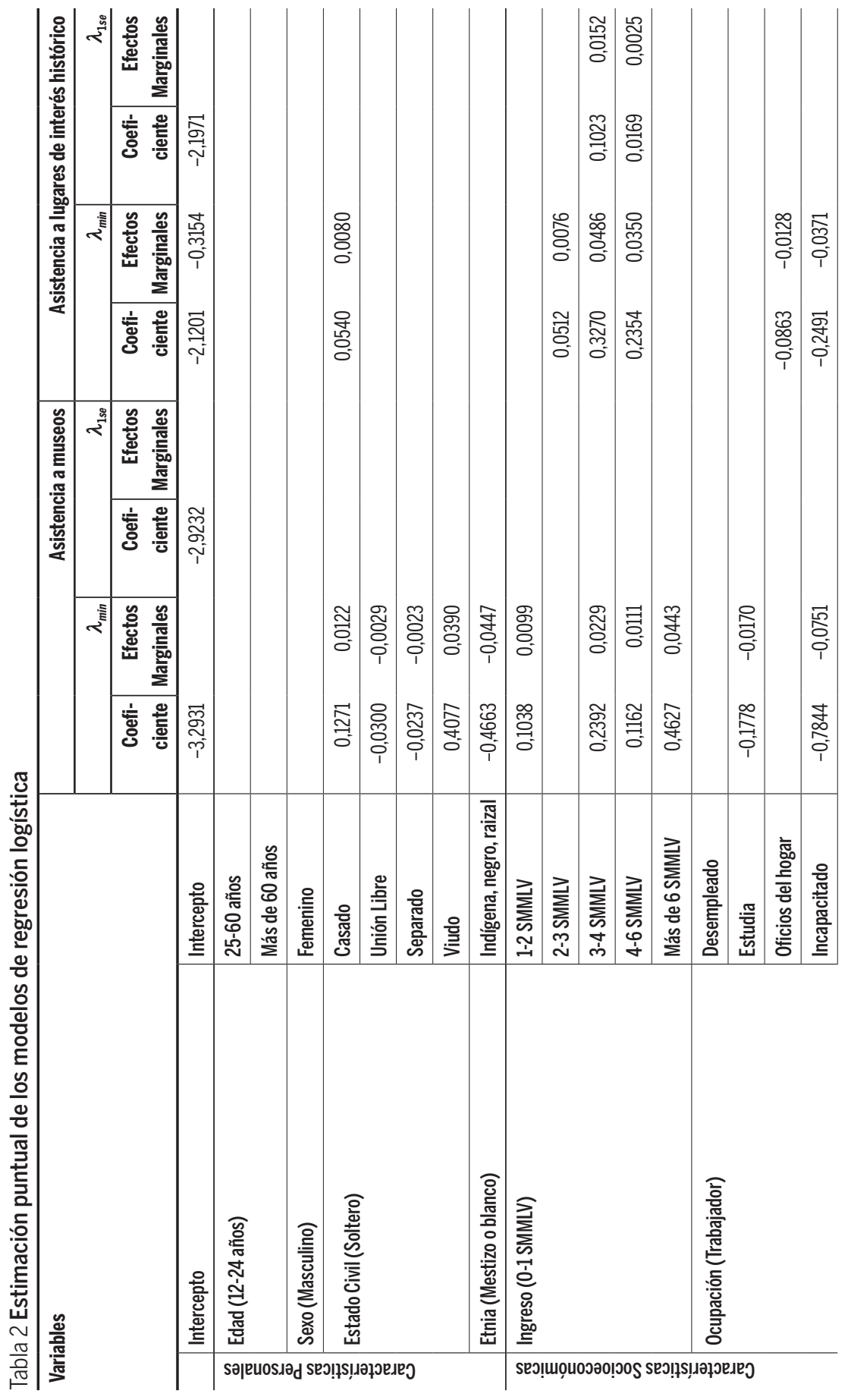




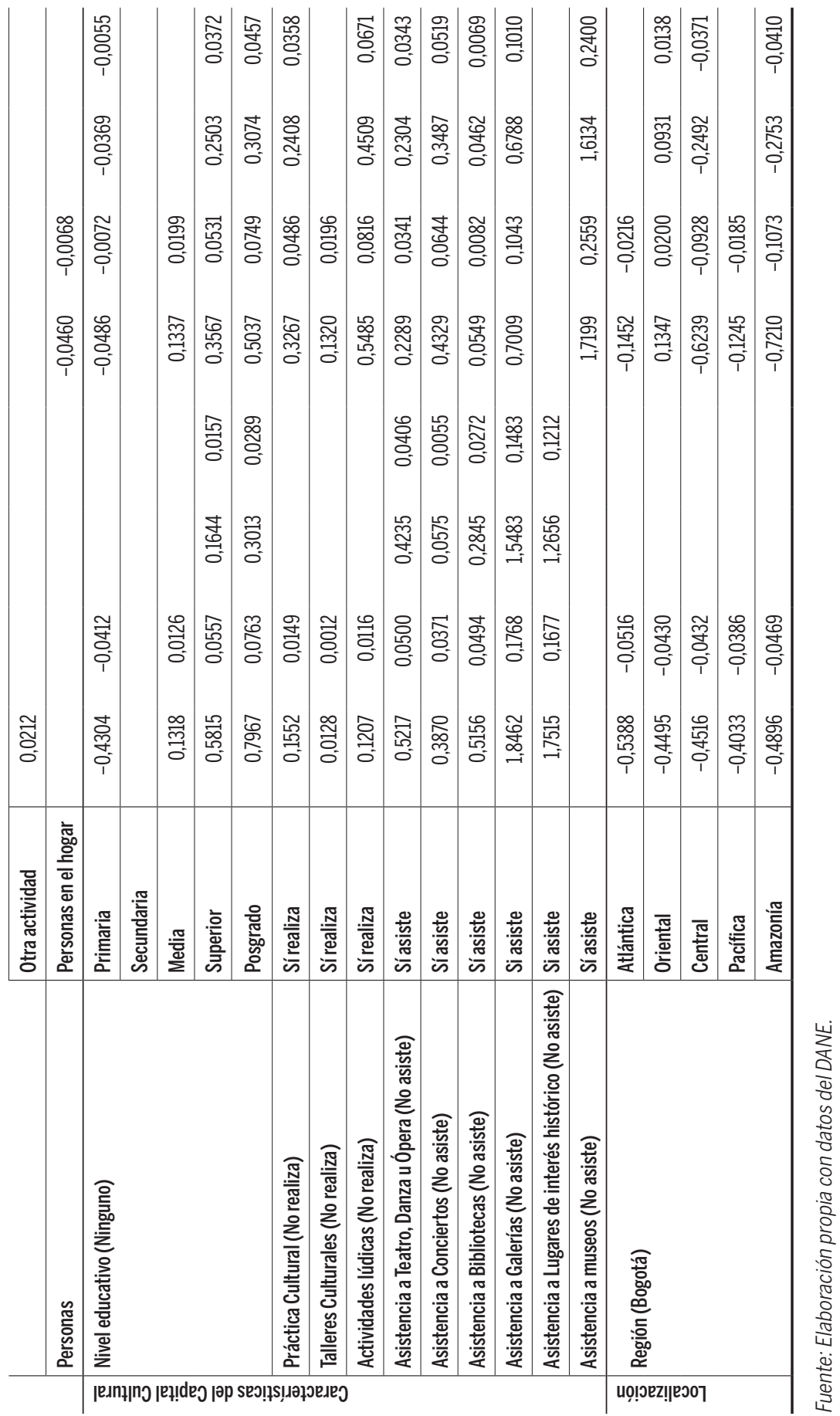


Precisamente los resultados muestran que el nivel de ingresos es relevante en los dos modelos y refleja las restricciones monetarias de los individuos para atender los gastos en que incurren durante las visitas (entradas, costos de transporte y otros gastos relacionados). Con respecto a quienes devengan entre 0-1 SMMLV, los individuos con un nivel de ingresos medio/alto (superior a 3 SMMLV) tienen mayor probabilidad de asistir a museos. Los efectos marginales son de 2,29\% para los que ganan entre 3-4 SMMLV, $1,11 \%$ para los que ganan entre 4-6 SMMLV y 4,43\% para los que ganan más de $6 \mathrm{SMMLV}$. La probabilidad de asistencia a lugares de interés histórico, por su parte, es más alta en los niveles intermedios de ingreso (entre 2-6 SMMLV), siendo la categoría entre 3-4 SMMLV la que muestra el mayor efecto marginal (4,86\%). Estos resultados indican que existen diferencias en el efecto del nivel de ingreso sobre la participación en las actividades analizadas. Mientras la asistencia a museos una actividad realizada por individuos de ingresos altos, la asistencia a lugares de interés histórico es realizada por individuos con ingreso medio. Al analizar la importancia general de esta variable, se observa que el mayor efecto marginal es similar para ambas actividades (4,43\% para museos y $4,86 \%$ para lugares de interés histórico).

Debido a que la educación y los ingresos resultaron relevantes, es interesante establecer cuál de estas variables es más importante para determinar la asistencia a las actividades consideradas. La comparación de los efectos marginales muestra que tanto para la asistencia a museos como para la asistencia a lugares de interés histórico, la magnitud del efecto marginal más alto del nivel educativo es superior al del efecto marginal más alto del nivel de ingresos, lo que indica que la educación del individuo es mejor predictor de la participación cultural que el ingreso. Este hallazgo también es validado empíricamente por Borgonovi (2004), Ateca-Amestoy (2009), Falk y Katz-Gerro (2016), entre otros.

La variable región de residencia revela hallazgos interesantes en los dos modelos. En el caso de la asistencia a museos, se observa que con respecto a Bogotá, las personas que residen en las regiones Atlántica, Central, Pacífica, Oriental y Amazonía, tienen una menor probabilidad de asistencia, siendo las regiones Atlántica y Amazonía las que tienen el efecto marginal más alto $(-5,16 \%$ y $-4,69 \%)$. Estas discrepancias regionales pueden explicarse por diferencias en la oferta, especialmente en las principales zonas urbanas como Bogotá que cuentan con una mayor dotación y variedad 
temática de estos espacios. En efecto, según el Sistema de Información de Museos Colombianos (SIMCO), en 2014 existían en Colombia 725 museos ubicados en 250 municipios de 31 departamentos; el 13,24\% de estos museos estaban ubicados en Bogotá, el 6,62\% en Medellín y el 4,13\% en Cali, es decir, la cuarta parte de los museos en el territorio se concentran en las tres ciudades principales. En el caso de la asistencia a lugares de interés histórico se observa un resultado similar. Con respecto a quienes viven en Bogotá, los residentes de las regiones Atlántica, Central, Pacífica y Amazonía tienen menor probabilidad de asistir, siendo el efecto marginal de las regiones Amazonía (-10,73\%) y Central (-9,28\%) los más altos.

Las características personales Edad y Sexo no resultaron relevantes en ninguno de los modelos y por tanto los coeficientes asociados son exactamente iguales a cero. Las responsabilidades familiares aproximadas por el Estado civil solo son relevantes para explicar la asistencia a museos, aunque al analizar los efectos marginales se observa éstos son muy cercanos a cero para todas las categorías, a excepción del estado civil viudo cuyo efecto marginal es de 3,9\% con respecto a los solteros. Las personas que se reconocen como miembros de una etnia (negros, indígenas, rom, gitanos) tienen un 4,4\% menos de probabilidad de asistencia a museos con respecto a los mestizos y blancos; este hallazgo refleja las diferencias en el acceso a estos bienes por parte de las comunidades étnicas que se explica por la no existencia de una oferta de museos que atienda a las demandas de los diferentes grupos étnicos. En lo referente a la ocupación, se observa que con respecto a los trabajadores, los incapacitados tienen 7,51\% menos de probabilidad de asistir a museos y $3,71 \%$ menos de asistir a lugares de interés histórico. Este hallazgo muestra que aunque los incapacitados tienen mayor disponibilidad de tiempo para asignar al consumo de actividades culturales, existen otro tipo de restricciones (como la movilidad) que dificultan su desplazamiento hasta el sitio específico de consumo.

Para identificar los determinantes con la mayor influencia en la decisión de participación, se hace más riguroso el proceso de selección de variables al considerar el parámetro de regularización $\lambda_{1 s e}$. En este escenario el número de variables relevantes en ambos modelos se reduce, es decir, se obtiene un modelo más parsimonioso aunque el costo que se asume es el incremento del error de clasificación en una desviación estándar. Los resultados que se presentan en la Tabla 2 refuerzan la idea de que el capital cultural es el principal determinante de la participación en las dos 
actividades analizadas: los dos niveles más altos del nivel educativo y la asistencia a otras actividades culturales, ambas variables relacionadas con el capital cultural de individuo, siguen siendo los determinantes más importantes tanto de la asistencia a museos como a lugares de interés histórico. Es importante notar que éstas variables son las únicas relevantes en el modelo para los museos (las demás tienen coeficientes asociados iguales a cero), lo que indica que el capital cultural es el determinante principal del consumo de esta actividad, por encima del ingreso. En el caso de la asistencia a lugares de interés histórico persisten adicionalmente los efectos del nivel de ingresos, las prácticas culturales, las actividades lúdicas y la región de residencia.

Con respecto al CRM estimado para la frecuencia de asistencia, en la Figura 3 y en la Figura 4 se presentan respectivamente los resultados del proceso de selección del parámetro de regularización óptimo. En el caso de la frecuencia de asistencia a museos se observa que al considerar el valor de $\lambda_{A I C}$, el número de variables relevantes es de 24 , aunque la trayectoria de los coeficientes converge rápidamente a cero, al punto que cuando la penalización se hace más rigurosa y se considera el $\lambda_{B I C}$ ninguna variable es relevante. Por esta razón se considera adicionalmente un tercer valor óptimo para el cual el BIC tiene un mínimo local $\left(\lambda_{B I C 2}\right)$ y el número de variables relevantes es de 4 . Un comportamiento similar se identifica en el modelo para la frecuencia de asistencia a lugares de interés histórico: el número de variables relevantes seleccionadas con base en el parámetro $\lambda_{\text {AIC }}$ es de 17 aunque los coeficientes se acercan rápidamente a cero a medida que la penalidad se hace más rigurosa, de modo que cuando se considera el $\lambda_{B I C}$ el número de variables relevantes es de solamente 2 . En la Tabla 3 se presenta la estimación puntual de cada uno de los coeficientes para cada valor óptimo del parámetro de regularización.

Los resultados, considerando el parámetro de regularización $\lambda_{A I C}$, indican que la práctica activa y la asistencia a otras actividades del sector cultural tienen un efecto positivo en la intensidad con que los individuos asisten a museos, es decir, no solamente existe una relación de complementariedad en la decisión de asistencia a las actividades consideradas, sino que también existe una relación positiva en la frecuencia con que se realiza el consumo. La región de residencia también es relevante aunque el signo de las categorías es negativo, es decir, con respecto a Bogotá, quienes residen en otras regiones del país asisten a museos con menor frecuencia, 
aspecto que refleja el efecto de la mayor dotación de museos en la capital del país. Por otra parte, aunque las categorías asociadas al ingreso y al nivel educativo tienen magnitudes diferentes de cero, no se identifica un efecto consistente con lo que se esperaría desde la teoría económica. Concretamente, con respecto a quienes no tienen educación, quienes tienen Secundaria y Media asiste con menor frecuencia, mientras que para los que tienen estudios de educación superior el efecto es positivo. En el caso del ingreso, solamente los individuos que ganan más de 6 SMMLV tienen una probabilidad mayor de asistir más frecuentemente con referencia a los que ganan menos de 1 SMMLV.

Figura 3 Selección del parámetro de regularización en el CRM (Museos)
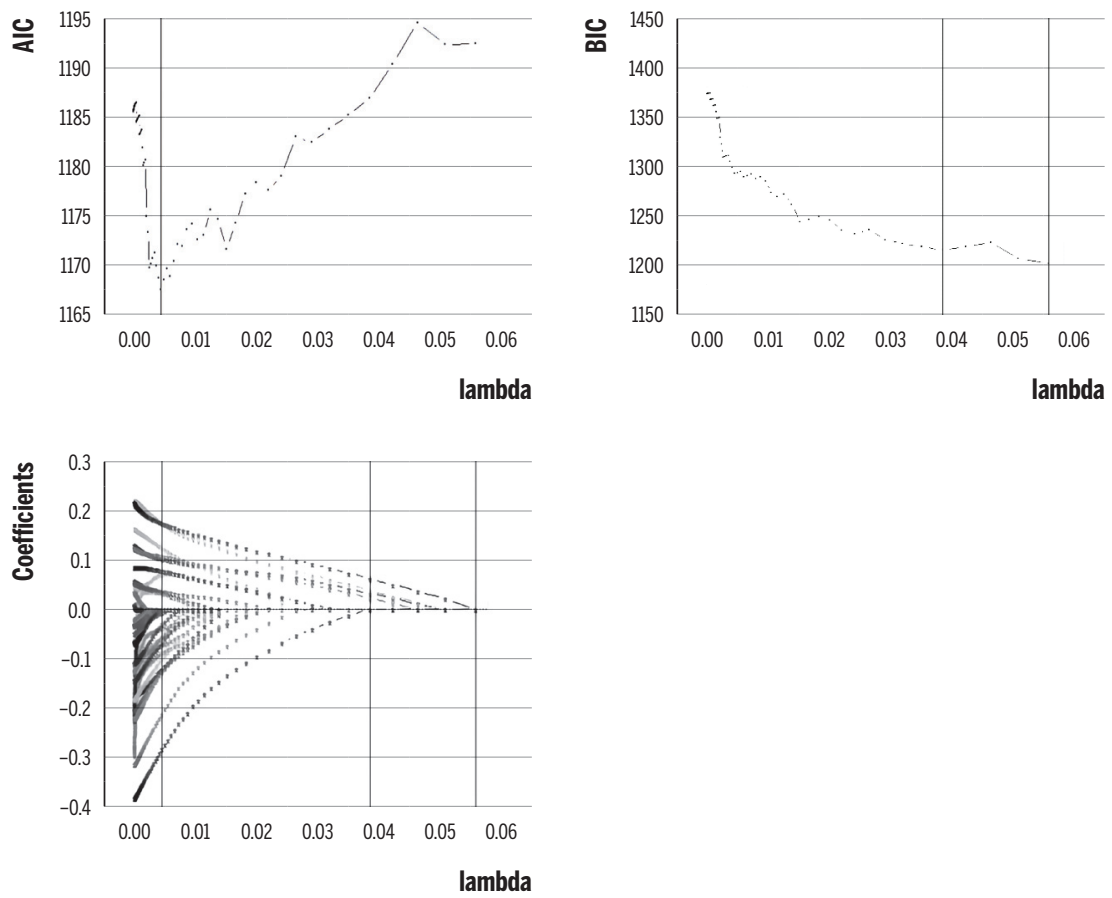

Fuente: Elaboración propia.

En el modelo para la frecuencia de asistencia a lugares de interés histórico se observa que la experiencia activa en prácticas culturales, talleres culturales y actividades lúdicas, es la dimensión del capital cultural con efecto positivo y relevante sobre la intensidad con que los individuos realizan las 


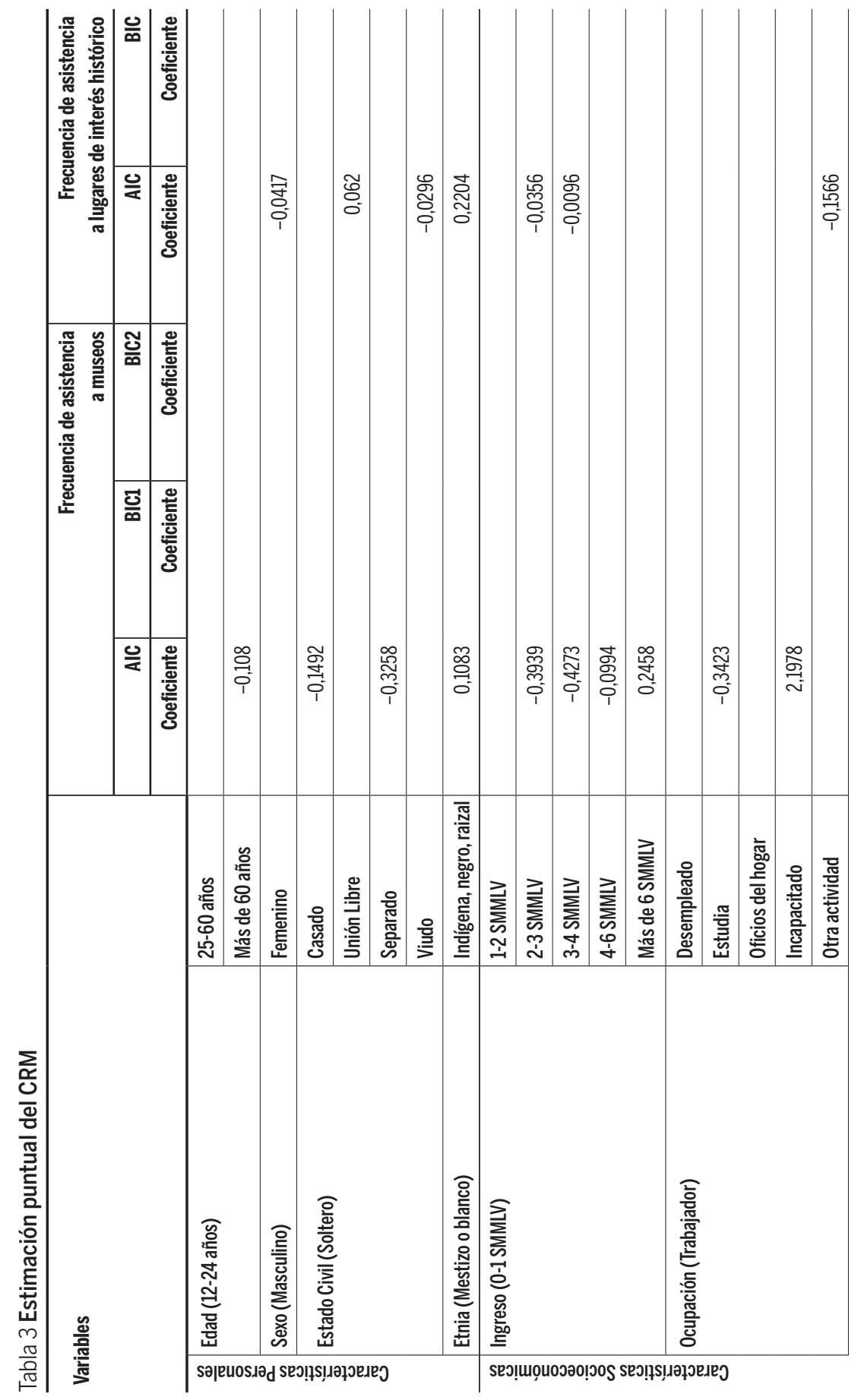




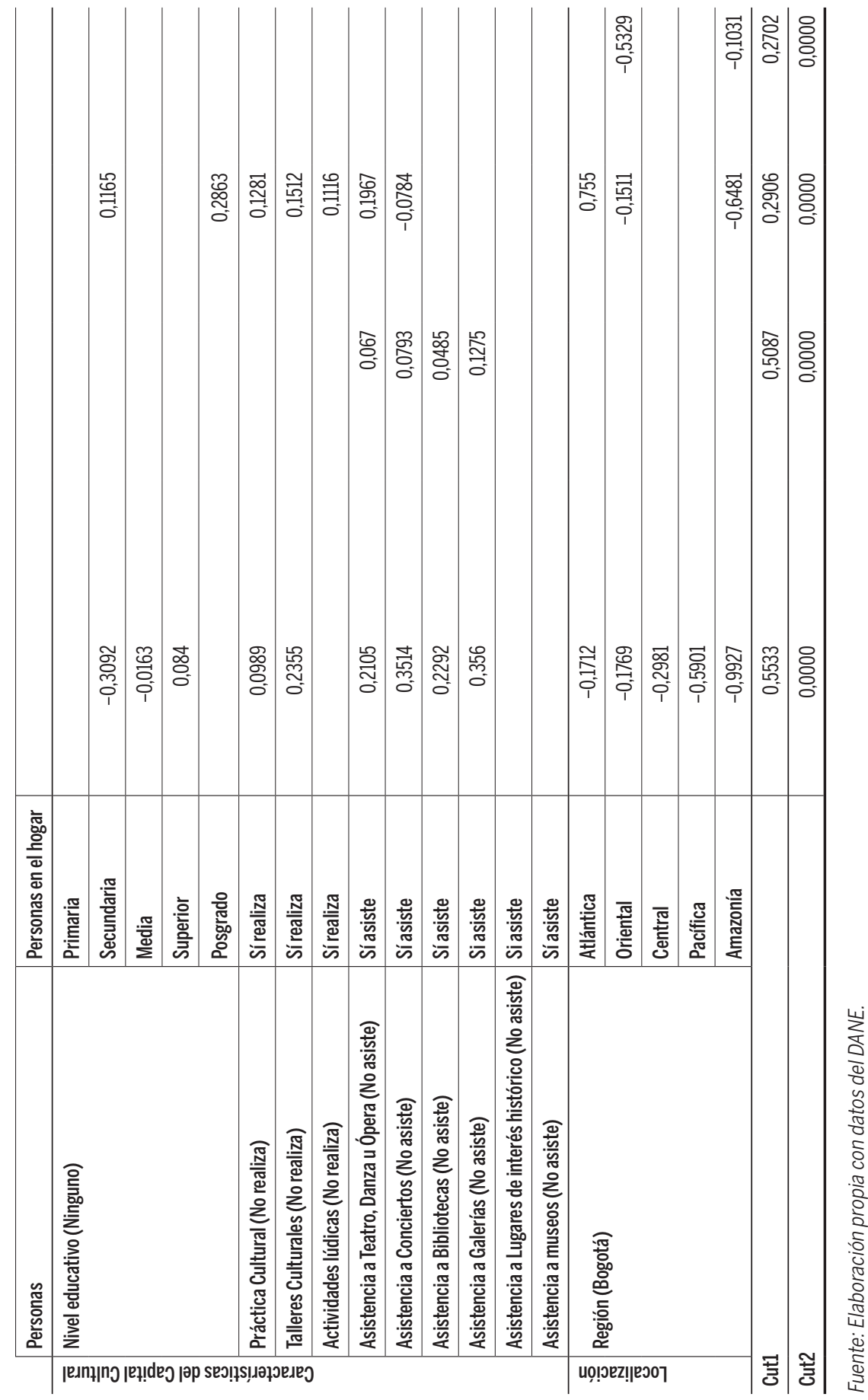


visitas; además, la asistencia a teatros es la única actividad cultural relacionada positivamente con la frecuencia de asistencia. En el caso de la región de residencia se observa que con respecto a quienes viven en Bogotá, los residentes en la región Atlántica tienen una probabilidad más alta de asistir con mayor frecuencia a lugares históricos, mientras que para quienes viven en la región Oriental y en la región Amazonía esta probabilidad es menor. Es importante notar que similar a lo observado en la frecuencia de asistencia a museos, no se obtuvo un efecto consistente con la teoría económica del nivel educativo ni del nivel de ingresos; en efecto, solamente las personas con Secundaria y Posgrado asisten con mayor frecuencia a lugares de interés histórico, mientras que para los que ganan entre 2-4 SMMLV este efecto es negativo.

Figura 4 Selección del parámetro de regularización en el CRM (Sitios de interés histórico)
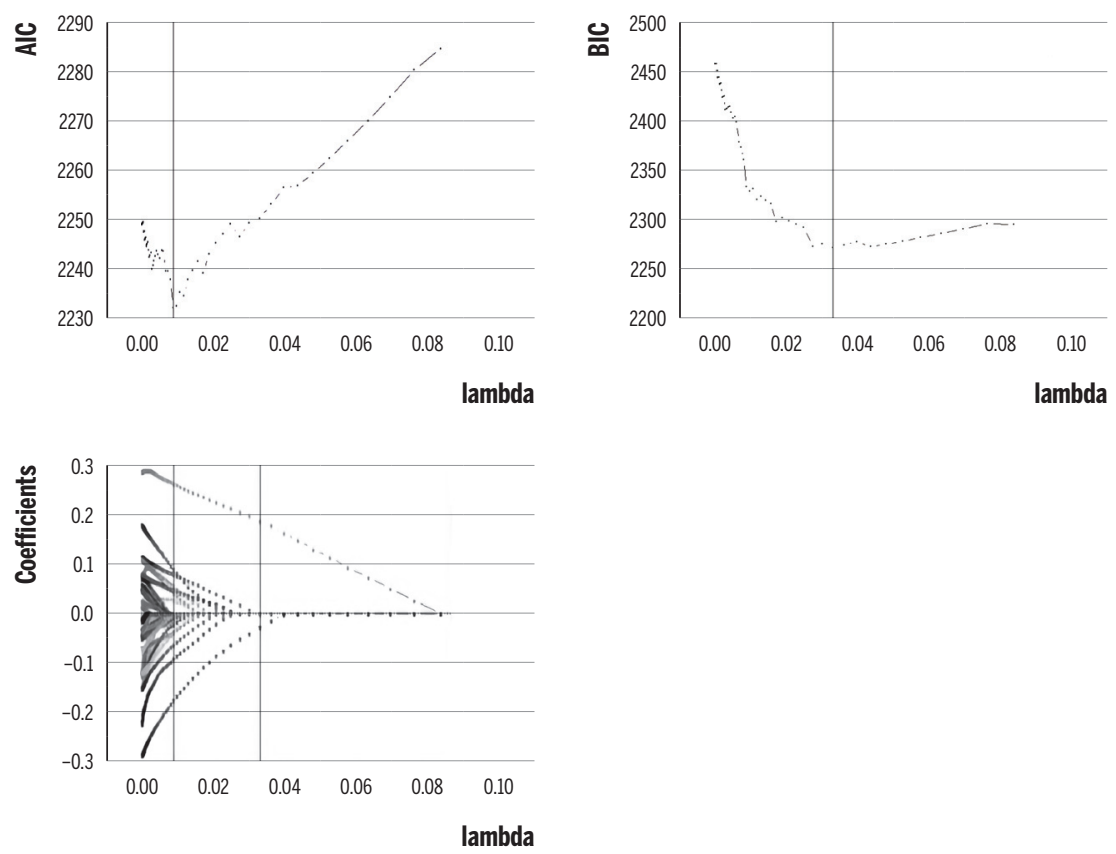

Fuente: Elaboración propia.

Cuando se considera los parámetros de regularización $\lambda_{B I C} \circ \lambda_{B I C 2}$ y el proceso de selección de variables es más riguroso, se identifican las varia- 
bles explicativas más importantes para determinar la frecuencia de asistencia a las actividades analizadas. En el caso de la asistencia a museos, se observa que solamente la asistencia a teatro, conciertos, bibliotecas y galerías de arte, relacionadas con el capital cultural del individuo, son las que mantienen efectos relevantes y positivos sobre la intensidad del consumo, mientras que todas las demás variables explicativas tienen un coeficiente asociado diferente de cero. Este hallazgo es similar al reportado por Fernández-Blanco y Prieto-Rodríguez (2009) y Wen y Cheng (2013) quienes encontraron que el capital cultural acumulado a través de las experiencias de consumo es un componente fundamental en la definición de las preferencias y de la capacidad de apreciación del consumo de actividades culturales a través del tiempo; además, este componente tiene un mayor efecto sobre la intensidad del consumo que incluso las características personales, el nivel de ingresos y el nivel educativo. En el caso de la asistencia a lugares de interés histórico, solamente persisten efectos de la región de residencia, mientras que las demás variables dejan de ser relevantes.

\section{Discusión y conclusiones}

En el presente artículo se analizaron los determinantes de la asistencia y frecuencia de asistencia a museos y lugares de interés histórico en Colombia, utilizando los microdatos de la ECC aplicada por el DANE en 2014. El marco teórico sobre el que se fundamenta este trabajo es el de la adicción racional (Stigler y Becker, 1977; Becker y Murphy, 1988) según el cual, las decisiones de consumo de un bien cultural dependen fundamentalmente de la capacidad de apreciación de la que dispone el individuo y que a su vez está condicionada por la acumulación de capital cultural. La disponibilidad de información permitió aproximar el capital cultural en este estudio por el nivel educativo, la experiencia práctica en actividades lúdicas, talleres culturales o prácticas culturales, y la asistencia como espectador a otro tipo de eventos culturales.

El enfoque metodológico adoptado fue la regularización de modelos de elección discreta: logit regularizado para la decisión de asistencia y CRM para la frecuencia de asistencia. Este enfoque se eligió teniendo en cuenta los problemas estadísticos que se presentan en los modelos tradicionales 
cuando el número de variables explicativas es alto como pérdida de parsimonia, inestabilidad, imprecisiones estadísticas, dificultad en la interpretación, pérdida de la capacidad de predicción y clasificación, multicolinealidad incidental, entre otros. La penalidad impuesta sobre el negativo de la función de la verosimilitud fue la Elastic Net, que se obtiene como la combinación lineal convexa de la penalidad Ridge y la penalidad Lasso. De esta forma es posible reducir la magnitud de los coeficientes estimados y seleccionar las variables realmente relevantes en el problema de investigación simultáneamente.

Los resultados indican que en Colombia existe una brecha en la participación cultural en las dos actividades analizadas debido a que la tasa de asistencia es muy baja (10,72\% para museos y $18,18 \%$ para sitios de interés histórico), y a que la mayoría de los asistentes son ocasionales. Una vez se controla por las características socioeconómicas de los individuos, es posible afirmar que el capital cultural es el principal determinante del consumo cultural por encima del nivel de ingreso, hallazgo que valida empíricamente el cumplimiento del enfoque de la adicción racional en el contexto colombiano. Coherente con esta idea, en el análisis de las razones de no asistencia se observó que el desinterés, consecuencia de un bajo nivel de capital cultural acumulado, es el principal motivo por el cual los individuos no asisten a estas dos actividades, por encima de la falta de dinero. En el caso de Colombia, este resultado es el esperado debido a que el Estado realiza una importante inversión en las políticas y programas culturales de formación de públicos y apropiación del patrimonio cultural que tienen como objetivo garantizar que las restricciones monetarias no sean una barrera para la participación.

Aunque la asistencia a museos y sitios de interés histórico compite a la hora de asignar el tiempo de ocio con el resto de actividades culturales como teatro, galerías de arte, conciertos o bibliotecas, estas actividades no entran en conflicto con ninguna actividad cultural activa o pasiva, sino que resulta complementaria a todas ellas; esto permite inferir que los asistentes a museos son consumidores culturales omnívoros pues están abiertos al consumo de diferentes tipos de arte y el espectro de actividades culturales entre las cuales distribuyen el consumo es más amplio y variado (Chan y Goldthorpe, 2007). En una futura investigación este sería un tema interesante de abordar para profundizar en la comprensión del comportamiento del consumidor cultural colombiano. 
En los anteriores resultados se advierte un importante papel para una política cultural asertiva que contribuya a la disminución de las brechas de participación cultural en Colombia. Es necesario que las políticas y programas de fomento al consumo cultural vayan más allá de la financiación de entradas. Las diferencias observadas en la asistencia a los bienes del patrimonio cultural responden fundamentalmente a diferencias en los niveles de capital cultural acumulado que no permite que un segmento importante de la población pueda desarrollar las habilidades cognitivas necesarias para descifrar los contenidos simbólicos que el patrimonio cultural incorpora. Fortalecer los programas de formación y creación cultural, especialmente en las edades tempranas, podría ser una estrategia de política para que la población tenga un acercamiento práctico y didáctico a las actividades culturales que contribuya al proceso de formación del gusto por esta actividad. Claramente, el tipo de programas que se realicen dependerá de la actividad que se quiera fomentar, sea museos o sitios de interés histórico.

Este trabajo constituye un aporte a la literatura aplicada sobre participación cultural en el país y en la región. Revisada la literatura son escasas las contribuciones en este tema y de las pocas que se identificaron, ninguna ha sido aplicada al caso de los museos y lugares de interés histórico. Otro aporte de este trabajo es la propuesta de la utilización de una metodología estadística novedosa que no ha sido empleada hasta el momento en la modelación de las decisiones de participación cultural, como son los modelos de elección discreta regularizados. Adicionalmente, la estimación del CRM para la frecuencia de asistencia es un aporte a la modelación de este tipo de comportamientos, pues el carácter progresivo en la intensidad del consumo cultural como resultado del proceso de formación del gusto es incorporado desde la misma definición de la variable de interés.

Finalmente, es necesario llamar la atención en que si bien es cierto que las mediciones de las decisiones de consumo cultural en Colombia son recientes y pueden tener efectos en la formulación de políticas culturales, es necesario construir información más precisa que permita analizar de una forma más profunda el consumo de bienes y servicios culturales e el país. Como resultado de esta investigación se impone solicitarle al DANE que su encuesta indague por otras variables que permitan capturar mejor el efecto del capital cultural del individuo sobre la participación cultural o verificar el cumplimiento de los diferentes enfoques teóricos en el contexto colombiano. Entre los aspectos a incluir en la encuesta se propone: 
educación artística y cultural diferenciada por edades de comienzo, si algún miembro de la familia pertenece al sector artístico, nivel de educación formal de los padres, experiencias de consumo previas, satisfacción de las expectativas en las experiencias de consumo previo y costos incurridos en cada visita.

\section{Referencias}

AGUADO, L.; PALMA, L. Determinants of performing arts participation in Colombia. En: $17^{\text {th }}$ International Conference on Cultural Economics by the ACEI, Kyoto, 2012.

ARCHER, K.; HOU, J.; ZHOU, Q.; FERBER, K.; LAYNE, J.; GENTRY, A. ordinalgmifs: An R package for ordinal regression in high-dimensional data settings. Cancer informatics, v.13, p.187, 2014.

ATECA-AMESTOY, V. Determining heterogeneous behavior for theater attendance. Journal of Cultural Economics, v.32, n.2, p.127-151, 2008.

ATECA-AMESTOY, V. El capital humano como determinante del consumo cultural. Estudios de Economía Aplicada, v.27, n.1, p.87-110, 2009.

BECKER, G.; MURPHY, K. A theory of rational addiction. Journal of Political Economy, v.96, n.4, p.675-700, 1988.

BENNETT, T.; BUSTAMANTE, M.; FROW, J. The Australian space of lifestyles in comparative perspective. Journal of Sociology, v.49, n.(2-3), p.224-255, 2013.

BERMÚDEZ, J.; MEDINA, L.; AGUADO, L. La decisión de escuchar música grabada en Colombia. Un enfoque microeconométrico. Revista de Métodos Cuantitativos para la Economía y la Empresa, v.21, p.21-38, 2016.

BORGONOVI, F. Performing Arts: An Economic Approach. Applied Economics, v.36, p.1871$1885,2004$.

BOURDIEU, P. Distinction: A social critique of the judgment of taste. London: Routledge, 1984.

BRIDA, J.; NOGARE, C.; SCUDERI, R. Frequency of museum attendance: motivation matters. Journal of Cultural Economics, v.40, n.3, p.261-283, 2016.

BÜHLMANN, P.; VAN DER GEER, S. Statistics for High-Dimensional Data. Springer books, 2011.

CAMERON, A.; TRIVEDI, P. Microeconometrics: methods and applications. Cambridge University Press, 2005.

CHAN, T.; GOLDTHORPE, J. The social stratification of theatre, dance and cinema attendance. Cultural Trends, v.14, n.3, p.193-212, 2005.

CHAN, T.; GOLDTHORPE, J. Social stratification and cultural consumption: Music in England. European Sociological Review, v.23, n.1, v.1-19, 2007.

DEVESA, M.; HERRERO, L.; SANZ, J. Análisis económico de la demanda de un festival cultural. Estudios de Economía Aplicada, v.27, n.1, p.139-160, 2009. 
DANE (2014). Ficha Metodológica, Encuesta de Consumo Cultural. Informe de Resultados.

DINIZ, S.; MACHADO, A. Analysis of the consumption of artistic-cultural goods and services in Brazil. Journal of Cultural Economics, v.35, n.1, p.1-18, 2011.

EVERETT, M.; BARRETT, M. Investigating sustained visitor/museum relationships: Employing narrative research in the field of museum visitor studies. Visitor Studies, v.1, n.1, p.2-15, 2009.

FALK, M.; KATZ-GERRO, T. Cultural participation in Europe: Can we identify common determinants? Journal of Cultural Economics, v.40, n.2, p.127-162, 2016.

FAN, J.; HAN, F.; LIU, H. Challenges of big data analysis. National Science Review, v.1, n.2, p.293-314, 2014.

FAVARO, D.; FRATESCHI, C. A discrete choice model of consumption of cultural goods: The case of music. Journal of Cultural Economics, v.31, n.3, p.205-234, 2007.

FERNÁNDEZ-BLANCO, V.; PRIETO-RODRÍGUEZ, J. Análisis de los hábitos de lectura como una decisión económica. Estudios de Economía Aplicada, v.27, n.1, p.87-110, 2009.

FRIEDMAN, J.; HASTIE, T.; TIBSHIRANI, R. Regularization paths for generalized linear models via coordinate descent. Journal of statistical software, v.33, n.1, p.1-22, 2010.

GALLÓN, S.; VÁSQUEZ, J. Aplicación de la Teoría de la Clasificación al Problema del Abandono Estudiantil: Un Estudio de Caso. En: Cuarta Conferencia Latinoamericana sobre el Abandono en la Educación Superior CLABES, 2014.

GÓMEZ-HERNÁNDEZ, L.Y.; ESPINAL, N.E. Determinantes de la participación en artes escénicas en Medellín, Colombia. Ensayos de Economía, v.2, n.48, p.189, 2016.

GUERZONI, M.; NUCCIO, M. Music consumption at the dawn of the music industry: the rise of a cultural fad. Journal of Cultural Economics, v.38, n.2, p.145-171, 2014.

HARDIN, J.: HILBE, J.; HILBE, J. Generalized linear models and extensions. Stata press, 2007.

HASTIE, T.; TIBSHIRANI, R. y FRIEDMAN, J. The Elements of Statistical Learning: Data Mining, Inference, and Prediction. Springer, 2009.

HASTIE, T.; TIBSHIRANI, R.; WAINWRIGHT, M. Statistical learning with sparsity. CRC press, 2015.

HENSHER, D.; ROSE, J.; GREENE, W. Applied choice analysis: a primer. Cambridge University Press, 2005.

HOU, J.; ARCHER, K. Regularization method for predicting an ordinal response using longitudinal high-dimensional genomic data. Statistical applications in genetics and molecular biology, v.14, n.1, p.93-111, 2015.

MACHADO, A.; GOLGHER, A.; DINIZ, S.; GAMA, L. Consumption of cultural goods and services and time allocation in Brazil. Nova Economia, v.27, n.1, p.35-63, 2017.

MONTORO-PONS, J.; CUADRADO-GARCÍA, M.; CASASÚS-ESTELLÉS, T. Analysing the popular music audience: Determinants of participation and frequency of attendance. International Journal of Music Business Research, v.2, n.1, p.35-62, 2013.

MUÑIZ, C.; RODRÍGUEZ, P.; SUÁREZ, M. Participation in cultural activities: specification issues. Journal of Cultural Economics, v.41, n.1, p.71-93, 2017. 
NOTTEN, N.; LANCEE, B.; VAN DE WERFHORST, H.; GANZEBOOM, H. Educational stratification in cultural participation: cognitive competence or status motivation? Journal of Cultural Economics, v.39, n.2, p.177-203, 2015

PALMA, L.; PALMA, L.; AGUADO, L. Determinants of cultural and popular celebration attendance: the case study of Seville Spring Fiestas. Journal of Cultural Economics, v.37, n.1, p.87-107, 2013.

PALMA, L.; AGUADO, L.; OSORIO, A. Determinants of book reading and library attendance in Colombia. A microeconometric approach. Economics and Business Letters, v.3, n.2, p.79-86, 2014.

STIGLER, G.; BECKER, G. De gustibus non est disputandum. The American Economic Review, v.67, n.2, p.76-90, 1977.

WEN, W.; CHENG, T. Performing arts attendance in Taiwan: who and how often? Journal of Cultural Economics, v.37, n.2, p.309-325, 2013.

\section{Sobre los autores}

Andrey David Ramos Ramírez-adramosr@unal.edu.co

Universidad Nacional de Colombia, Medellín, Colombia.

ORCID: https://orcid.org/0000-0002-5795-3190.

Nora Elena Espinal Monsalve - nespinal@unal.edu.co

Universidad Nacional de Colombia, Medellín, Colombia.

ORCID: https://orcid.org/0000-0003-2777-7410.

\section{Sobre el articulo}

Artículo recibido el 02 de febrero de 2018. Aceptado para publicación el 04 de febrero de 2019. 